\title{
PROPERTIES OF RANK PRESERVING WEAK MAPS
}

\author{
BY DEAN LUCAS
}

Communicated by Gian Carlo Rota, April 16, 1973

Let $P_{X}$ be the set of all combinatorial pregeometries (or matroids) [5] on the $n$-set $X . P_{X}$ is partially ordered by $G \rightarrow H$ (in lieu of $G \geqq H$ ) whenever the identity mapping from $G$ to $H$ is weak (i.e. each independent set of $H$ is independent in $G$ ). A map which preserves geometric rank will be written $G \stackrel{*}{\longrightarrow} H$. A map $G \rightarrow H$ is called simple if $G$ covers $H$ in the partial order on $P_{X}$.

Any map, $G \rightarrow H$, has a decomposition into simple maps (just select a saturated chain between $G$ and $H$ ). In general such a decomposition will consist of some simple maps which are rank preserving and others which reduce rank (see Figure 1). Rank reducing simple maps are always rank one upper truncations (in the language of Crapo [4], a truncation, $G \rightarrow H$, is simple iff $G$ is a co-atom in the lattice of erections of $H$ ). A result of Kennedy [6] shows that the truncation of any erectable pregeometry is never simple. Thus any weak map is decomposed into an alternating sequence of upper truncations and rank preserving weak maps. Upper truncations and their properties are well understood. The object of this research is to study the properties of rank preserving weak maps.

The following lemma collects some preliminary facts which are easily proved by checking independent sets.

LEMma 1. For $G$ and $H$ in $P_{X}, G \stackrel{*}{\longrightarrow} H$, and $p$ in $X$, if $G / p$ denotes the contraction of $G$ by $p, G-p$ the deletion of $p$ from $G$, and $\widetilde{G}$ the Whitney dual of $G([\mathbf{1}]$ or $[\mathbf{5}])$, then

(a) $G / p \stackrel{*}{\longrightarrow} H / p$ provided $p$ is not a loop of $H$.

(b) $G-p \stackrel{*}{\longrightarrow} H-p$ provided $p$ is not an isthmus of $H$.

(c) $\tilde{G} \stackrel{*}{\longrightarrow} \tilde{H}$.

Any minor $M=\left(G / A^{\prime}\right)-B^{\prime}$ of $G$ can be expressed as $M=(G / A)-B$ in which no loop is contracted and no isthmus deleted. In Proposition 1 and Corollaries 1 and 6 we assume that the indicated minors are expressed in this manner.

AMS (MOS) subject classifications (1970). Primary 05B35, 05B25; Secondary 05A20, 15A03, 15A33, 50D30.

Key words and phrases. Weak map, simple weak map, weak map decomposition, combinatorial pregeometry, Tutte polynomial, binary geometry, matroid. 

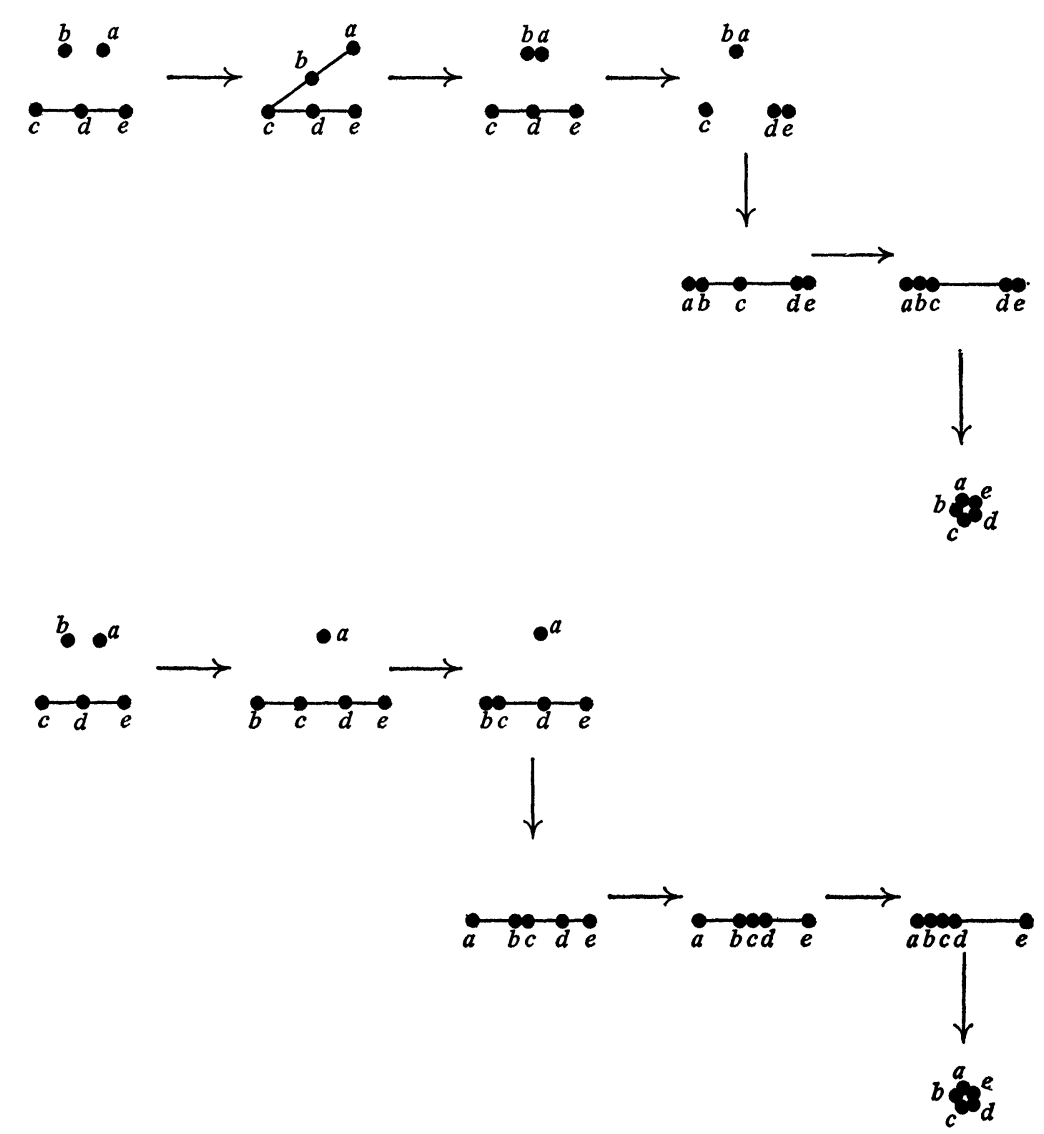

FIGURE 1. Two decompositions of the map Horizontal maps are rank preserving, vertical maps are rank reducing.

Proposition 1. Let $G \stackrel{*}{\longrightarrow} H$. If $M=(H \mid A)-B$ is a minor of $H$ and $N=(G \mid A)-B$ is the corresponding minor of $G$ then $N \stackrel{*}{\longrightarrow} M$.

A geometry of rank $k$ is called free if all its $k$ element subsets are bases. If $F$ is free then $G \stackrel{*}{\longrightarrow} F$ iff $G=F$. This combined with Proposition 1 gives:

Corollary 1. If $G \stackrel{*}{\longrightarrow} H$ and $F=(H \mid A)-B$ is a free minor of $H$ then the corresponding minor, $(G \mid A)-B$, of $G$ is equal to $F$.

An important corollary now follows from the fact that a four point line is free and is the unique forbidden minor for binary pregeometries. 
COROLLARY 2. The image of a binary geometry under a rank preserving weak map is binary.

Let $P_{X, k}$ be the subset of $P_{X}$ consisting of all the pregeometries of rank $k$ along with the induced order. Corollary 2 says that the binary pregeometries of rank $k$ on $X$ form an order ideal in $P_{X, k}$. The following two theorems and their corollaries give a fairly complete description of the properties of weak maps within the binary ideal of $P_{X, k}$. The proofs involve observing the effect of rank preserving weak maps on matrices of zeros and ones.

THEOREM 1. If $G$ is binary and $G \stackrel{*}{\longrightarrow} H$ with $G \neq H$ then $H$ is separable.

THEOREM 2. ' Let \#G be the number of connected components in a complete direct sum decomposition of $G$. If $G$ is binary and $G \stackrel{*}{\longrightarrow} H$ and $\# H>\# G+1$ then there exists $L$ in $P_{X}$ with $\# L=\# H-1$ and $G \stackrel{*}{\longrightarrow} L \stackrel{*}{\longrightarrow} H$.

COROLlary 3. If $G$ is binary then a map $G \stackrel{*}{\longrightarrow} H$ is simple iff \#H= $\# G+1$.

COROllary 4. If $G$ is binary and $H$ is a simple image of $G$ of the same rank then $H=(G / F) \oplus F$ for some subgeometry $F$ of $G$.

COROLlaRY 5. Within the binary ideal of $P_{X, k}$ the chain condition holds, the length of a saturated $G, H$ chain being \#H-\#G.

Proposition 1 and Theorem 1 combine to provide

COROllary 6. If $G$ is binary and $G \stackrel{*}{\longrightarrow} H$ and $M=(H \mid A)-B$ is a connected minor of $H$ then the corresponding minor, $(G / A)-B$, of $G$ is equal to $M$.

The celebrated representation theorems of Tutte [8] together with Corollary 6 provide three corollaries which show that some well-known subclasses of geometries are well behaved under rank preserving weak maps.

Corollary 7. If $G \stackrel{*}{\longrightarrow} H$ and $G$ is unimodular then so is $H$.

COROLlaRY 8. If $G \stackrel{*}{\longrightarrow} H$ and $G$ is graphical then so is $H$.

Corollary 9. If $G \stackrel{*}{\longrightarrow} H$ and $G$ is planar graphical then so is $H$.

The final corollary is useful in the theory of bracket rings [9] where it supports the conjecture that the bracket ring of a unimodular geometry is an integral domain. The proof follows from Corollary 4.

COROLlaRY 10. Suppose $G$ is a binary pregeometry of rank $k$ on $a$ $2 k$-set $X$ and $B_{1}, X-B_{1}, B_{2}, X-B_{2}$ are all bases of $G$. If $G \stackrel{*}{\longrightarrow} H$ then $B_{1}, X-B_{1}$ are both bases of $H$ iff $B_{2}, X-B_{2}$ are both bases of $H$. 
If $G$ is not binary then Theorem 1 and Corollaries 3, 4, 5, 6, and 10 do not hold. In connection with Corollaries 2 and 7 it should be noted that coordinatizability over fields of characteristic other than two is not preserved by rank preserving weak maps. It is conjectured, however, that Theorem 2 is true in the absence of the binary condition (hence if $G \stackrel{*}{\longrightarrow} H$ is simple then $\# H \leqq \# G+1)$.

Most of what can be said about rank preserving weak maps in the general case involves $t(G)$, the Tutte polynomial of the pregeometry $G$. This concept was introduced by Crapo [3] and studied extensively by Brylawski [1]. The following two theorems are proved by induction on $|X|$.

THEOREM 3. If $G \stackrel{*}{\longrightarrow} H$ then $\left.t(G)\right|_{z, 0} \geqq\left. t(H)\right|_{z, 0}$ for all $z>0$. Further, the inequality is strict if $G$ is loopless and $G \neq H$.

Evaluations at $z<0$ (hence colorability and critical exponent values) are not so well behaved.

THEOREM 4. If $G \stackrel{*}{\longrightarrow} H$ then

$$
\left.\frac{\partial^{k} t(G)}{\partial z^{k}}\right|_{z, 0} \geqq\left.\frac{\partial^{k} t(H)}{\partial z^{k}}\right|_{z, 0} \text { for all } z \geqq 0 \text { and positive integers } k \text {. }
$$

Part (c) of Lemma 1 provides

COROLlaRY 11. Theorems 3 and 4 also hold for evaluations at $(0, x)$ with $x \geqq 0$ (where the derivative of Theorem 4 is taken with respect to $x$ ).

Since many interesting geometric invariants are evaluations of the Tutte polynomial or its derivatives, it follows from Theorems 3 and 4 that these invariants are altered systematically by rank preserving weak maps. The next corollaries give two significant examples.

CoROLLARY 12. If $G \stackrel{*}{\longrightarrow} H$ then $|\mu(G)| \geqq|\mu(H)|$ with strict inequality if $G$ is loopless and $G \neq H(\mu(G)=\mu(0,1)$ of the lattice of flats of $G$ as defined by Rota [7]).

COROLlaRY 13. If $G \stackrel{*}{\longrightarrow} H$ then $\beta(G) \geqq \beta(H)(\beta(G)$ is the invariant studied by Crapo in [2]).

From Corollary 13 it follows (and is easy to prove directly) that the image of a separable pregeometry under a rank preserving weak map is also separable.

All the above results are also true if the symbol $G \longrightarrow H$ is taken to mean there exists a weak rank preserving bijection between representatives of the isomorphism classes of $G$ and $H$. 


\section{BIBLIOGRAPHY}

1. T. H. Brylawski, A decomposition for combinatorial geometries, Trans. Amer. Math. Soc. 171 (1972), 235-282.

2. H. H. Crapo, A higher invariant for matroids, J. Combinatorial Theory 2 (1967), 406-417. MR 35 \#6579.

3. —, The Tutte polynomial, Aequationes Math. 3 (1969), 211-229. MR 41 \#6705.

4. - Erecting geometries, Proc. Second Chapel Hill Conf. on Combinatorial Math. and Appl., Univ. North Carolina, Chapel Hill, N.C., 1970, pp. 74-99. MR 42 \#7536.

5. H. H. Crapo and G.-C. Rota, Combinatorial geometries, M.I.T. Press, Cambridge, Mass., 1970 (preliminary ed.).

6. D. Kennedy, private correspondence.

7. G.-C. Rota, On the foundations of combinatorial theory. I. Theory of Möbius functions, Z. Wahrscheinlichkeitstheorie und Verw. Gebiete 2 (1964), 340-368. MR 30 \#4688.

8. W. T. Tutte, Lectures on matroids, J. Res. Nat. Bur. Standards Sect. B 69B (1965), 1-47, MR 31 \#4023.

9. N. White, The bracket ring and combinatorial geometry, Ph.D. Thesis, Harvard University, Cambridge, Mass., 1971.

Department of Mathematics, University of North Carolina, Chapel Hill, NORTH CAROLINA 27514 